\title{
CADEIA PRODUTIVA DE ENERGIA DE BIOMASSA NA REGIÃO DO PLANALTO SUL DE SANTA CATARINA: UMA ABORDAGEM PROSPECTIVA ${ }^{1}$
}

\author{
Flávio José Simioni² e Vitor Afonso Hoeflich ${ }^{3}$
}

\begin{abstract}
RESUMO - Objetivou-se, neste estudo, identificar e prospectar o comportamento futuro dos fatores críticos de desempenho da cadeia produtiva de energia a partir de biomassa de origem florestal, bem como as demandas de capacitação e de pesquisas. O estudo teve como área de abrangência os municípios da região de Lages, no Planalto Sul de Santa Catarina. Utilizou-se como metodologia a abordagem de prospecção foresight, desenvolvida através da projeção de especialistas com base no seu próprio conhecimento, mediante a aplicação de questionário Delphi, contemplando os elos da produção florestal e da indústria e a geração de energia. As principais conclusões foram: a) há disponibilidade de resíduos florestais para uso na geração de energia, sendo necessária a readequação do processo de colheita florestal; b) investimentos na qualificação de recursos humanos e em novas tecnologias nos processos industriais são requeridos para melhor aproveitar os recursos florestais; e c) tende a haver aumento do uso da biomassa de origem florestal para a produção de energia.
\end{abstract}

Palavras-chave: Energia, Prospecção e Demandas.

\section{PRODUCTIVE CHAIN OF BIOMASS ENERGY IN THE SOUTH PLATEAU AREA LOCATED IN SANTA CATARINA: A PROSPECTIVE APPROACH}

\begin{abstract}
This paper aims to identify and prospect the future behavior of a critical fact about the biomass energy productive chain in an original forest, as well as to check the needs of training and research demands to improve its competitive performance. The study had as its inclusion area the municipal districts of the area of Lages, in the South Plateau in Santa Catarina. Foresight approach was the methodology used, developed through the specialists projection based on their knowledge, by applying a Delphi questionnaire, and contemplating the segments of the forest production, industry and generation of energy. The main conclusions were: a) readiness of forest residues exists to use for the production of energy, it has been necessary to adapt the process in forest crop; b) investments and qualification in human resources and new industrial technology processes are required for taking better advantage of forest resources; and c) the production of energy by using the biomass in the original forest might grow.
\end{abstract}

Keywords: Energy, Search and Demands.

\section{INTRODUÇÃO}

A região do Planalto Sul de Santa Catarina tem no agronegócio florestal sua principal atividade econômica. Fazem parte deste várias cadeias produtivas, como a de madeira sólida, celulose e papel, móveis e energia que, juntamente com outras atividades econômicas complementares, a exemplo da indústria de máquinas e equipamentos para madeira e a estrutura de colheita e transporte florestal, constituem uma aglomeração industrial chamada por alguns autores de cluster da madeira (HOFF e SIMIONI, 2004).

Como resultado da concentração de plantios florestais e da atividade industrial, significativos volumes de resíduos de biomassa são gerados, desde o processo de colheita florestal, bem como nas diferentes fases do processo de transformação da madeira. Brand e Neves

\footnotetext{
${ }^{1}$ Recebido em 14.08.2008 e aceito para publicação em 25.08.2010.

${ }^{2}$ Universidade do Estado de Santa Catarina, UDESC, Brasil. E-mail: <simioni@uniplac.net>.

${ }^{3}$ Universidade Federal do Paraná, UFPR, Brasil.
} 
(2005), estudando a quantificação e qualificação dos resíduos da indústria de base florestal na região de Lages, em 2005, chegaram à cifra de 180 mil toneladas/mês.

Parte do resíduo é utilizada pelas próprias empresas que o produzem para a geração de energia necessária aos processos industriais, como na geração de vapor para a secagem de madeira, de papel ou outros fins. Porém, até o início da década de 2000 grande volume desse material não tinha uso específico, dando origem, assim, à poluição ambiental, principalmente da água e do ar, devido à sua queima a céu aberto ou à autocombustão provocada pela estocagem inadequada.

Diante dessa disponibilidade de biomassa e da crescente busca de fontes de energia alternativas, com menor impacto ambiental que os combustíveis fósseis, novos investimentos passaram a ser realizados na região visando à produção de energia através de usinas de cogeração. Paralelamente, as fontes tradicionais de energia térmica (óleo combustível e gás) foram, por parte das indústrias locais, parcialmente substituídas por biomassa de origem florestal (resíduos da colheita florestal e da indústria). Assim, os resíduos deixaram de ser vistos como "lixo" e passaram a serem tratados como matéria-prima.

Essa nova configuração, na qual a biomassa de origem florestal passou a ser mais disputada, provocou alterações nos processos de concorrência, sobretudo nas estratégias adotadas para a garantia de suprimento (SIMIONI et al., 2007a). Essas transformações no mercado de biomassa necessitam ser estudadas, visando orientar a tomada de decisões e a proposição de políticas públicas e privadas para o planejamento estratégico regional do setor, no intuito de buscar o seu crescimento e desenvolvimento sustentável.

Diante desse cenário, a agricultura energética desponta como grande oportunidade para atender às necessidades de fontes de energia renovável, principalmente a biomassa (GOVERNO, 2005). Segundo o Balanço Energético Nacional (BRASIL, 2003), a participação da biomassa na matriz energética brasileira é de $27 \%$, a partir da utilização de lenha de carvão vegetal (11,9\%), bagaço de cana-de-açúcar $(12,6 \%)$ e outros (2,5\%). Conforme Parikka (2004), cerca de dois quintos do potencial de biomassa no mundo são usados e, em muitos locais, a biomassa corrente é utilizada abaixo do potencial disponível.
De acordo com Couto et al. (2002, p. 1), “a biomassa de origem florestal é uma forma de energia limpa, renovável, equilibrada com o meio ambiente rural e urbano, descentralizadora de população, geradora de empregos (tanto no meio rural como no meio urbano) e criadora de tecnologia própria”. Paralelamente, avança significativamente a busca pela certificação, promovendo o uso ambientalmente correto dos recursos florestais (JACOVINE et al., 2006). Brand et al. (2004) e Brand (2007) destacaram também que, além da alta disponibilidade da biomassa florestal atualmente no Brasil, são gerados muitos resíduos na indústria que podem ser aproveitados para geração de energia. No âmbito das indústrias, a utilização do material gerado nos processos industriais pode viabilizar a implantação de sistemas de cogeração, aumentando a potencialidade de geração de energia a partir de biomassa (BONDUELLE et al., 2003).

A utilização de fontes renováveis de geração de energia com tecnologias que contribuem para o uso eficiente e limpo tem forte tendência no cenário internacional. Conforme apontou o relatório sobre o estado da arte e tendências das tecnologias para energia (MACEDO, 2003), o desenvolvimento de P\&D na direção de tecnologias que conferem maior sustentabilidade ambiental é priorizado no mundo todo. Nesse aspecto, o relatório aponta a biomassa energética como área de interesse, pois apresenta custos bastante interessantes e competitivos internacionalmente. Além desse aspecto, a disponibilidade de áreas para plantios de florestas é muito grande. Ainda de acordo com o relatório, os riscos associados ao suprimento e aos impactos ao meio ambiente farão crescer o interesse em combustíveis “limpos”, em que a biomassa deverá suprir uma fração crescente da demanda de energia. Estudos realizados pelo Centro de Gestão e Estudos Estratégicos (CGEE) em prospecção tecnológica em energia apontam que as tecnologias relacionadas ao uso de biomassa para geração de energia deverão ser objeto de novos estudos prospectivos (JANUZZI et al., 2004).

Assim, os objetivos desta pesquisa foram identificar e prospectar o comportamento futuro dos fatores críticos, bem como as demandas de capacitação e de pesquisas que visem ao melhor desempenho competitivo da cadeia produtiva de energia a partir de biomassa de origem florestal. O estudo foi realizado no Planalto Sul de Santa Catarina e considerou os elos da produção florestal, da indústria e da produção de energia a partir de biomassa. 


\section{METODOLOGIA}

A pesquisa teve como área de abrangência o Planalto Sul do Estado de Santa Catarina, considerando a região geográfica compreendida por uma distância de, aproximadamente, 120 km do Município de Lages, contemplando 24 municípios do seu entorno.

Para a realização da análise prospectiva da cadeia produtiva de energia a partir de biomassa florestal, utilizou-se o modelo de avaliação de cadeia produtiva proposto por Castro et al. (1998) e utilizado pela EMBRAPA na prospecção de demandas tecnológicas de cadeias produtivas. A abordagem empregada foi a do foresight, desenvolvido através da projeção de especialistas com base no seu próprio conhecimento e mediante a aplicação do questionário Delphi, com o intuito de subsidiar a formulação de políticas, o planejamento e a tomada de decisões.

Inicialmente, levando em conta sugestões apresentadas por Simioni e Hoff (2006) e Simioni et al. (2007b), foram identificados os fatores críticos ao desempenho da cadeia produtiva a partir de uma análise diagnóstica, os quais, por sua vez, foram priorizados e constituíram os objetos da análise prospectiva. De acordo com Castro et al. (1998) e Castro et al. (2002), os fatores críticos são entendidos como qualquer variável que afeta, de modo relevante, o desempenho em análise. Se o efeito for positivo, está-se diante de uma força propulsora; ao contrário, tem-se uma força restritiva.

O questionário Delphi foi elaborado a partir de Lima et al. (2005) e dividido em quatro partes, em que na primeira o especialista realizou uma autoavaliação, identificando seu nível de especialização e conhecimentos (perito ou especialista, conhecedor, familiarizado e não familiarizado) sobre os diferentes elos da cadeia produtiva. Nas etapas posteriores, abordaram-se questões sobre o elo da produção florestal, da indústria e do uso de biomassa para a geração de energia. Em cada elo foi avaliado o grau de influência dos fatores críticos, identificados e priorizados na fase diagnóstica, sobre o seu desempenho. O grau de influência variou em uma escala de 0 (zero) a 10, sendo zero para influência quase nula e 10 para influência extremamente elevada.

Conforme preconizaram Wright (1995) e Kupfer e Tigre (2004), o questionário Delphi foi enviado a 50 especialistas, ou seja, pessoas conhecedoras do objeto de estudo, com a finalidade de relatarem suas opiniões a respeito de questões que estão relacionadas à probabilidade de acontecimentos futuros. Tais especialistas foram intencionalmente selecionados, objetivando contemplar os diferentes agentes que atuam na cadeia, quais sejam: empresas, setor público, universidades, associações e sindicatos, pesquisadores, centros de treinamento, sistema legal e regulatório, entre outros. Houve o retorno de $46 \%$ dos questionários, ou seja, 23 questionários respondidos.

Para a realização da análise dos dados obtidos pela aplicação do questionário Delphi, foram consideradas, segundo Astigarraga (s.d.) e Lima et al. (2005), medidas que indicam a tendência central dos dados, quais sejam: a média do grau de influência, ponderada segundo a autoavaliação, considerando peso 4 para perito, 3 para conhecedor, 2 para familiarizado e 1 para não familiarizado; a mediana; o primeiro quartil (Q1) e o terceiro quartil (Q3).

A partir dos dados levantados pelo questionário Delphi, realizou-se um workshop, com reuniões de grupos e apresentação dos resultados em plenária. O objetivo do workshop foi apresentar os resultados dessa coleta de dados, a fim de validá-los mediante a análise e discussão das questões e buscar a formulação de estratégias, políticas e ações coletivas, visando enfrentar os fatores críticos à cadeia produtiva de energia. Foram convidadas a participar do evento as mesmas pessoas que responderam ao mencionado questionário. O workshop foi realizado com a presença de 18 (dezoito) participantes.

\section{RESULTADOS E DISCUSSÃO}

\subsection{A produção florestal}

A análise das informações (Tabela 1) permite observar que todos os fatores críticos, exceto a concentração dos plantios florestais, apresentaram tendência de aumento do grau de influência no futuro em relação ao período atual. A redução do peso da concentração dos plantios florestais pode ser explicada pelos crescentes investimentos em plantações florestais por parte de pequenas empresas e produtores autônomos e pela prática do fomento florestal utilizada de forma crescente na região.

Os fatores legislação ambiental e certificação florestal foram os que se mostraram com _maior elevação do grau de influência. Esses fatores apresentam alta correlação, uma vez que para a obtenção da certificação 
Tabela 1 - Grau de influência atual e futura dos principais fatores críticos à produção florestal na região do Planalto Sul de Santa Catarina.

Table 1 - Degree of current and future influence of the main critical factors to the forest production in the area of the South Plateau of Santa Catarina.

\begin{tabular}{|c|c|c|c|c|c|c|c|c|}
\hline \multirow[t]{2}{*}{ Fator crítico } & \multicolumn{4}{|c|}{ Influência atual } & \multicolumn{4}{|c|}{ Influência futura - 2020} \\
\hline & Média & Mediana & Q1 & Q3 & Média & Mediana & Q1 & Q3 \\
\hline Legislação ambiental & 7,6 & 7,0 & 6,3 & 8,8 & 9,3 & 10,0 & 9,0 & 10,0 \\
\hline Espécies florestais & 4,6 & 5,0 & 3,0 & 6,0 & 5,7 & 6,5 & 3,5 & 7,0 \\
\hline Manejo florestal & 5,4 & 5,0 & 5,0 & 6,8 & 5,8 & 6,5 & 3,0 & 8,0 \\
\hline Concentração dos plantios florestais & 6,9 & 8,0 & 6,0 & 8,0 & 6,4 & 8,0 & 5,0 & 8,0 \\
\hline $\begin{array}{l}\text { Pressões ambientais e sociais sobre a } \\
\text { cultura do Pinus }\end{array}$ & 6,8 & 7,0 & 5,0 & 8,0 & 7,9 & 8,5 & 6,3 & 10,0 \\
\hline Pragas e doenças & 5,8 & 6,0 & 5,0 & 7,0 & 6,5 & 6,0 & 5,0 & 9,0 \\
\hline Certificação florestal & 5,0 & 5,0 & 4,3 & 6,0 & 7,6 & 8,0 & 6,3 & 10,0 \\
\hline
\end{tabular}

Fonte: Elaborado pelos autores com base nos dados da coleta de campo.

Source: Authors based on data obtained in field data collection.

há a necessidade de atender à legislação vigente. De acordo com a pesquisa, 55,6\% dos entrevistados consideraram muito importante e $44,4 \%$ tinham como importante a certificação florestal, que tenderá sofrer aumento para aproximadamente $95 \%$ dos entrevistados. É interessante observar que as justificativas mencionadas pelos respondentes para o aumento da certificação florestal estão centradas no atendimento às normas de legislação e exigências de mercado, que serão adotadas pelos maiores plantadores de florestas. No entanto, a certificação dificilmente será alcançada pelos pequenos produtores, uma vez que estes apresentam dificuldades para atender às normas do sistema de certificação.

Considerando o processo de colheita florestal, 100\% dos entrevistados consideraram viável o uso múltiplo da floresta, no intuito de obter o aproveitamento dos resíduos (ponta de árvore, galhos e outras partes) para a geração de energia. Essa realidade é esperada para o ano de 2010. As necessidades de pesquisa para que essa prática seja possível são: a) identificação da quantidade e qualidade de biomassa florestal disponível na região; b) mercado regional de biomassa florestal; c) logística e custos de coleta e transporte de biomassa florestal; d) viabilidade econômica da colheita dos resíduos florestais; e) ciclagem de nutrientes em florestas com colheita dos resíduos florestais; f) disponibilidade futura de biomassa florestal; g) potencialidade de usos da biomassa; h) impactos ambientais da colheita de resíduos das florestas; e i) evolução do consumo de biomassa e produção e consumo de energia nas empresas.

Segundo a resposta de $44 \%$ dos entrevistados, a disponibilidade de madeira e de resíduos florestais tende a sofrer importante crescimento. Já, de acordo com 38,9\% dos respondentes, haverá elevação, porém não muito significativa. As principais justificativas estão associadas ao acréscimo da área plantada na região e à existência de grande volume de plantios. Os aspectos que mais limitam o crescimento são o aumento da rigidez da legislação e a falta de apoio à atividade.

Quando indagados a respeito de qual fator é mais importante na limitação do aumento da área plantada, cerca de $60 \%$ dos entrevistados indicaram a legislação florestal como o fator mais importante (Tabela 2). As justificativas, para tais afirmações, estão centradas, na sua maioria, no cunho preservacionista da legislação e na limitação do uso de áreas, bem como no aumento dos custos dos plantios florestais. Já a disponibilidade de espécies foi indicada por $43 \%$ dos profissionais amostrados como o fator menos importante, justificadas pelo fato de que as espécies disponíveis apresentam boa adaptação e altos índices de produtividade e de que novas espécies estão em desenvolvimento ou já estão sendo introduzidas na região.

As necessidades de pesquisa para o elo da produção florestal correspondem ao melhoramento genético de espécies nativas; viabilidade técnica e econômica de consorciação de espécies; potencial madeireiro das espécies de eucalipto; biotecnologia relacionada às florestas; sustentabilidade da produtividade em plantios florestais; impactos das florestas plantadas sobre o meio ambiente; e viabilidade de plantios florestais energéticos.

As ações indicadas na pesquisa no sentido de potencializar ou viabilizar o aproveitamento das oportunidades do segmento da produção florestal são: 
Tabela 2 - Grau de importância de fatores selecionados sobre a área plantada com florestas. Table 2 -Degree of importance of factors selected on the area planted with forests.

\begin{tabular}{lcc}
\hline Fator limitante & $(\%)$ mais importante & (\%) menos importante \\
\hline Legislação florestal & $\mathbf{5 7 , 9}$ & 0,0 \\
Preço da terra & 10,5 & 7,1 \\
Pressões sociais & 5,3 & 21,4 \\
Disponibilidade de espécies & 5,3 & $\mathbf{4 2 , 9}$ \\
Disponibilidade de terras & 15,8 & 28,6 \\
Outros & 5,3 & 0,0 \\
TOTAL & 100,0 & 100,0 \\
\hline
\end{tabular}

Fonte: Elaborado pelos autores com base nos dados da coleta de campo.

Source: Authors based on data obtained in field data collection.

incentivos à produção de florestas de qualidade em pequenas propriedades, pela ampliação da assistência técnica e linhas de crédito compatíveis; capacitação na área de produção de florestas, principalmente por meio de cursos técnicos e treinamentos; realização de diagnóstico florestal, visando fornecer informações sobre a base florestal atual para subsidiar um planejamento florestal de longo prazo; e criação de cooperativas, objetivando congregar pequenas e médias empresas para aumento da competitividade.

\subsection{A indústria}

Analisando os dados relativos à indústria (Tabela 3), verificou-se que, à exceção do licenciamento ambiental e nível de escolaridade dos funcionários, os demais fatores (cultura local extrativista, cultura local não associativa, padrão tecnológico, deficiência na gestão de processos, ausência de dados estatísticos do setor, planejamento estratégico regional e baixo valor agregado dos produtos) apresentaram tendência de ligeira queda ou de permanecer constante. Essas respostas podem ser explicadas, em parte, pela existência de ações que estão sendo desenvolvidas na região, as quais têm ação direta na diminuição desses fatores. A questão do licenciamento ambiental está, de acordo com a visão dos respondentes, diretamente relacionada com o aumento das exigências da legislação e com deficiências de recursos humanos e financeiros dos órgãos executores.

Quanto às ações a serem tomadas nas empresas, a totalidade dos entrevistados concorda que a qualificação profissional dos colaboradores é uma necessidade urgente para a indústria, uma vez que, além de apresentarem baixo nível de escolaridade, são deficientes em conhecimentos técnicos e específicos.

De modo geral, as principais ações sugeridas pelos entrevistados para o segmento indústria foram: cursos profissionalizantes relacionados à madeira; cursos relacionados à produção e inovações tecnológicas;

Tabela 3 - Grau de influência atual e futura dos principais fatores críticos na indústria na região do Planalto Sul de Santa Catarina.

Table 3 - Degree of current and future influence of the principal critical factors to the industry in the area of the South Plateau of Santa Catarina.

\begin{tabular}{|c|c|c|c|c|c|c|c|c|}
\hline \multirow[t]{2}{*}{ Fator crítico } & \multicolumn{4}{|c|}{ Influência atual } & \multicolumn{4}{|c|}{ Influência futura - 2020} \\
\hline & Média & Mediana & Q1 & Q3 & Média & Mediana & Q1 & Q3 \\
\hline Licença ambiental & 6,3 & 6,5 & 5,0 & 7,8 & 8,0 & 8,0 & 7,0 & 9,8 \\
\hline Nível de escolaridade dos funcionários & 7,0 & 7,0 & 5,0 & 8,0 & 7,2 & 8,0 & 6,0 & 9,0 \\
\hline Cultura local extrativista & 6,3 & 6,5 & 5,0 & 8,0 & 4,6 & 4,5 & 2,5 & 6,0 \\
\hline Cultura local não associativa & 7,9 & 8,0 & 7,0 & 8,8 & 7,5 & 7,5 & 6,0 & 8,0 \\
\hline Padrão tecnológico & 7,0 & 7,0 & 5,0 & 8,8 & 7,2 & 7,0 & 6,3 & 8,0 \\
\hline Deficiência na gestão de processos & 6,8 & 7,0 & 5,0 & 8,0 & 6,5 & 7,0 & 5,0 & 8,8 \\
\hline Ausência de dados estatísticos do setor & 6,5 & 7,0 & 5,0 & 8,0 & 6,2 & 6,0 & 5,0 & 7,8 \\
\hline Planejamento estratégico regional & 7,1 & 8,0 & 5,0 & 8,0 & 7,1 & 7,0 & 6,0 & 8,8 \\
\hline Baixo valor agregado dos produtos & 7,9 & 8,0 & 7,0 & 9,0 & 7,2 & 7,0 & 6,3 & 8,0 \\
\hline
\end{tabular}

Fonte: Elaborado pelos autores com base nos dados da coleta de campo.

Source: Authors based on data obtained in field data collection. 
parcerias entre empresas e universidades; planejamento estratégico regional; cursos de curta duração para processos industriais; fortalecimentos dos centros tecnológicos; cooperativismo e associativismo; desenvolvimento da área de design de móveis; viabilização de créditos para investimentos objetivando agregar valor à madeira; e políticas públicas para incentivar a implantação de indústrias moveleiras.

As demandas por pesquisas identificadas durante esse levantamento, para enfrentar os fatores críticos do segmento da indústria, foram: desenvolvimento de tecnologias para o aproveitamento da matéria-prima florestal e diversificação de produtos; identificação do padrão tecnológico utilizado na indústria, classificação da produção por estrato e por destino dos produtos; nível de qualificação dos recursos humanos e seu impacto sobre a competitividade; diagnóstico industrial dos diferentes segmentos da indústria na região; análise das diferentes técnicas de usinagem e rendimento de madeira serrada; desenvolvimento de processos industriais automatizados; tecnologia e otimização de processos industriais e do uso dos resíduos; desenvolvimento de processos de tratamento e preservação de madeiras competitivas; e análise dos potenciais de utilização da madeira na região.

A inclusão da cogeração de energia nas empresas de grande porte de forma integrada, com a possibilidade de direcionar a matéria-prima para energia ou para produtos finais, conforme as condições de mercado, é possibilidade concreta na opinião de 76,6\% dos entrevistados, indicando o ano 2012 em que essa realidade será amplamente difundida. Essa tendência é justificada pela crescente elevação dos preços de energia e pela possibilidade de uso múltiplo dos recursos florestais.
No que se refere aos novos investimentos no setor industrial, mais que $52 \%$ dos entrevistados consideraram que estes devem ser realizados nas empresas locais, mediante a diversificação e agregação de valor aos produtos, investimento tecnológico e cooperação entre empresas. Para cerca de $43 \%$ dos respondentes, os investimentos devem concentrar-se na área moveleira, justificado por duas razões: a deficiência desse segmento industrial na região e a possibilidade de agregação de valor à matéria-prima.

\subsection{A geração de energia}

Para todos os fatores críticos relativos a produção de energia de biomassa de origem florestal houve indicação de elevação do grau de influência no futuro, de forma consensual e com baixa dispersão das respostas (Tabela 4). Dessa maneira, os dados revelam, de um lado, que os fatores passarão a ter mais importância sobre a geração de energia e, de outro, o crescente mercado de biomassa na região. Chama atenção a significativa elevação da pontuação (32\% na média) dos fatores preço do petróleo e da energia elétrica, revelando preocupação com o fornecimento de energia onde a biomassa pode ser fonte alternativa para a geração de energia utilizada na produção industrial. A colheita florestal e o tratamento adequado dos resíduos também obtiveram significativo aumento da pontuação média (de 65\% e 43\%, respectivamente), indicando a preocupação com a quantidade e qualidade desses.

De acordo com 56\% dos entrevistados, a geração de energia a partir de resíduos de origem florestal sofrerá aumento considerável no futuro, e para 39\% deles, haverá aumento, porém não muito significativo. Essa

Tabela 4 - Grau de influência atual e futura dos principais fatores críticos na produção de energia a partir de resíduos de origem florestal na região do Planalto Sul de Santa Catarina.

Table4 - Degree of current and future influence of the principal critical factors to the production of energy of residues of forest origins in the area of the South Plateau of Santa Catarina.

\begin{tabular}{|c|c|c|c|c|c|c|c|c|}
\hline \multirow[t]{2}{*}{ Fator crítico } & \multicolumn{4}{|c|}{ Influência atual } & \multicolumn{4}{|c|}{ Influência futura - 2020} \\
\hline & Média & Mediana & Q1 & Q3 & Média & Mediana & Q1 & Q3 \\
\hline Rendimento na indústria & 7,2 & 6,5 & 5,0 & 8,0 & 7,5 & 8,0 & 6,0 & 8,8 \\
\hline Preço do petróleo & 6,6 & 7,0 & 5,0 & 8,0 & 8,6 & 9,0 & 8,0 & 10,0 \\
\hline Preço da energia elétrica & 6,5 & 6,5 & 5,3 & 7,8 & 8,6 & 9,0 & 8,0 & 10,0 \\
\hline Qualidade da madeira & 5,4 & 5,5 & 4,0 & 7,8 & 6,6 & 7,0 & 5,0 & 8,0 \\
\hline Colheita florestal & 5,1 & 5,0 & 4,3 & 5,8 & 8,4 & 8,0 & 8,0 & 8,8 \\
\hline Tratamento adequado aos resíduos & 5,8 & 6,0 & 5,0 & 7,0 & 8,3 & 8,0 & 7,3 & 9,8 \\
\hline Tecnologia de geração de energia & 5,8 & 6,0 & 4,0 & 7,0 & 7,5 & 8,0 & 6,0 & 9,0 \\
\hline
\end{tabular}

Fonte: Elaborado pelos autores com base nos dados da coleta de campo.

Source: Authors based on data obtained in field data collection.

Revista Árvore, Viçosa-MG, v.34, n.6, p.1091-1099, 2010 
tendência é explicada, primeiro, pela crescente demanda por energia por parte das empresas, e a energia gerada a partir de biomassa florestal pode ser alternativa viável. Um segundo aspecto é o aumento dos plantios florestais, gerando também incremento da quantidade de biomassa, seja da floresta, seja da indústria na forma de resíduos, que pode ser utilizada para a geração de energia, configurando-se em mais uma forma de aproveitamento dos recursos oriundos da floresta.

Na perspectiva de aumento da geração de energia, alguns fatores passam a ser importantes na utilização da biomassa como matéria-prima. Conforme indicado na Tabela 5, a disponibilidade de biomassa associada à qualidade constitui um dos fatores mais apontados na pesquisa como limitantes da sua utilização. Entretanto, como fator menos importante, a qualidade da biomassa recebeu a maior pontuação, todavia isso se refere às indicações dos produtores de resíduos das indústrias madeireiras não preocupadas com essa questão. Assim, a tecnologia de geração de energia passa a ser o fator menos importante, justificado pelo seu grau de maturidade disponível no mercado.

No que se refere à qualidade dos resíduos, os fatores mais importantes que comprometem sua qualidade para a geração de energia são o teor de umidade, a presença de impurezas e a classificação por tipo ou categoria, com 30\%, 29\% e $24 \%$ das respostas, respectivamente. O teor de umidade da biomassa está diretamente relacionado com o poder calorífico, e, quanto maior a umidade, menor o poder calorífico gerado. Isso implica utilização de um volume de biomassa maior para a geração de uma mesma quantidade de energia. Esse fator tem sido utilizado como critério na determinação do preço da biomassa. A presença de impurezas implica geração de volume maior de resíduos do processo de queima (teor de cinzas), gerando maior impacto ambiental devido à sua destinação. Por fim, a classificação por tipo ou categoria torna-se importante para a blendagem do combustível destinado à queima nas caldeiras.

Conforme as informações desta pesquisa, tem-se que, para a obtenção de biomassa com melhores características para uso na geração de energia, as serrarias e o processo de colheita florestal são os setores que mais necessitam de ações visando à qualificação dos fornecedores. Destaca-se a necessidade de profissionalização e conscientização deles no sentido de que os resíduos precisam ser tratados como matériaprima e não como lixo, sobretudo nos aspectos de coleta, classificação, armazenamento e transporte.

Em termos gerais, de acordo com os entrevistados, as necessidades de pesquisa para geração de energia a partir de resíduos de origem florestal na região são: adequação do sistema de colheita florestal visando ao aproveitamento dos resíduos; adaptação do sistema de geração de energia para otimização do uso da biomassa; avaliação dos diferentes sistemas de estocagem de biomassa; identificação das propriedades energéticas dos diferentes tipos de resíduos madeireiros; avaliação da oferta e demanda de resíduos na região; avaliação da demanda de energia nas empresas da região; análise técnico-econômica e financeira do uso de biomassa para pequenas unidades de geração de energia; viabilidade econômica da pré-industrialização de resíduos florestais e industriais madeireiros; avaliação do potencial de utilização dos resíduos do processo de queima; eficiência do uso da biomassa para geração de energia térmica e elétrica; logística do suprimento de biomassa; e impactos sociais, ambientais e econômicos da produção de energia de biomassa.

Tabela 5 - Nível de importância dos fatores limitantes ao uso dos resíduos de origem florestal para a geração de energia no Planalto Sul de Santa Catarina.

Tabela 5 - Level of importance of the restrictive factors of the use of the residues of forest origin for the generation of energy in the South Plateau of Santa Catarina.

\begin{tabular}{|c|c|c|}
\hline Fator limitante & (\%) mais importante & (\%) menos importante \\
\hline Preço do petróleo & 17,6 & 18,8 \\
\hline Custo da biomassa & 11,8 & 6,3 \\
\hline Tecnologia de geração de energia a partir da biomassa & 5,9 & 25,0 \\
\hline Qualidade da biomassa & 29,4 & 31,3 \\
\hline Disponibilidade de biomassa & 35,3 & 18,8 \\
\hline TOTAL & 100,0 & 100,0 \\
\hline
\end{tabular}

Fonte: Elaborado pelos autores com base nos dados da coleta de campo.

Source: Authors based on data obtained in field data collection. 
Considerando essas observações, os resultados desta pesquisa também foram evidenciados por Bacchi (2008), que destacou que esforços devem ser feitos para desenvolver tecnologia que permita obter matériaprima de forma sustentável; maiores investimentos em $\mathrm{P} \& \mathrm{D}$, visando ao aumento da produtividade agrícola e industrial; melhoria da infraestrutura para escoamento dos produtos; capacitação técnica e científica de recursos humanos; e desenvolvimento de tecnologias para aproveitamento de resíduos.

\section{CONCLUSÃO}

A análise atual e a prospecção futura do grau de influência dos fatores críticos permitiram identificar necessidades, sobretudo de pesquisas, de capacitação e de ações coletivas, que poderiam promover melhor desempenho da cadeia estudada. Pelos resultados, pode-se inferir que essa tenha sido uma contribuição aplicada do estudo, a partir da qual gestores públicos e privados poderão subsidiar-se para a elaboração de suas ações estratégicas.

No que tange à produção florestal, destaca-se a importância dada para as questões relacionadas à legislação ambiental, o que denota a existência de conflitos entre o setor produtivo e o ambiente institucional constituído. Outro aspecto é a necessidade de reorganização do processo de colheita florestal, visando ao aproveitamento dos resíduos florestais para a produção de energia.

No elo da indústria, evidenciou-se a necessidade de qualificação dos recursos humanos e investimentos em tecnologias, visando ao uso mais eficiente dos recursos florestais. Essas medidas impactaram, de um lado, na redução do potencial de geração de resíduos industriais e, de outro, no tratamento e uso adequados desses resíduos.

A geração de energia a partir de biomassa de origem florestal apresenta tendência de elevação, muito determinada pelas circunstâncias de mercado dos combustíveis tradicionais e da disponibilidade dessa matéria-prima na região. Além da quantidade, a qualidade da biomassa, sobretudo nos aspectos de teor de umidade e classificação por tipo ou categoria, também é importante.

As demandas identificadas neste trabalho levam a inferir que ação ampla deve ser desenvolvida envolvendo todos os segmentos que formam o agronegócio florestal, objetivando a realização de planejamento de abrangência regional. Este deverá basear-se, fundamentalmente, na realização de um diagnóstico da base florestal e industrial da região e na definição de estratégias de médio e longo prazos.

\section{REFERÊNCIAS}

ASTIGARRAGA, E. El método delphi.

Donosita, San Sebastián:Universidad de Deusto, 2006. Acesso em: < www.codesyntax.com/prospectiva/ Metodo_delphi.pdf> Acesso em: 10 ago. 2006.

BACCHI, M. R. P. Brasil - gerando energia de biomassa, limpa e renovável. CEPEA. Disponível em: <http:www.cepea.esalq.usp.br> Acesso em: 21 julho/ 2008.

BONDUELLE, A.; YAMAJI, F. M.; BORGES, C. C. Resíduo de pinus: uma fonte para novos produtos. Remade, 2003. Disponível em: <http:// www.remade.com.br>. Acesso em: 28 set. 2004.

BRAND, M. A. et al. Avaliação do processo produtivo de uma indústria de manufatura de painéis por meio do balanço de material e do rendimento da matéria-prima. Revista Árvore, v.28, n.4, p.553-562, 2004. Disponível em: <http:// www.scielo.br/scielo.php>. Acesso em: 20 fev. 2008.

BRAND, M. A.; NEVES, M. D. Levantamento da disponibilidade dos resíduos industriais e florestais de madeira e avaliação da variação de sua qualidade energética em função das condições climáticas anuais, na região de Lages - Santa Catarina. Lages: UNIPLAC: Tractebel Energia, 2005. 73 p. (Relatório de Pesquisa)

BRAND, M. A. Geração de energia: o futuro da biomassa florestal. In: BINOTTO, E. (Org.). Cadeias produtivas e processos agroindustriais. Passo Fundo: UPF, 2007.

BRASIL. Ministério de Minas e Energia.

Balanço energético nacional 2003.

Brasília: SEM/MME, 2003. Disponível em: <http:// ftp.mme.gov.br/Pub/Balanco/BEN/Portugues/ Benp99.pdf.> Acesso em: 21 out. 2004.

CASTRO, A. M. G.; LIMA, S. M. V.; CRISTO, C. M. P. N. Cadeia produtiva: marco conceitual para apoiar a prospecção tecnológica. In: SIMPÓSIO DE GESTÃO DE TECNOLOGIA, 22., 2002, Salvador. Anais... São Paulo: FEA/USP, 2002. p.1-14. 
CAStro, A. M. G. et al. Prospecção tecnológica de cadeias produtivas e sistemas naturais. Brasília: Embrapa, 1998. 568p.

COUTO, L.; MÜLLER, M. D.; TSUKAMOTO FILHO, A. A. Florestas plantadas para energia: aspectos técnicos, sócio-econômicos e ambientais. In: CONFERÊNCIA -

SUSTENTABILIDADE NA GERAÇÃO E USO DE ENERGIA NO BRASIL: OS PRÓXIMOS VINTE

ANOS, 2002. Campinas: UNICAMP, 2002

Disponível em: <http:www.cgu.unicamp.br/ energia2020> Acesso em: 15 dez. 2006.

GOVERNO lança Plano Nacional de Agroenergia. Agroanalysis: Revista de Agronegócios, v.25, n.10, p.34-35, 2005.

HOFF, D. N.; SIMIONI, F. J. O setor de base florestal na serra catarinense. Lages: UNIPLAC, 2004. 268p.

JACOVINE, L. A. G. et al. Processo de implementação da certificação florestal nas empresas moveleiras nacionais. Revista Árvore, v.30, n.6, p.961-968, 2006. Disponível em: <http://www.scielo.br/scielo.php $>$. Acesso em: 20 fev. 2008.

JANNUZZI, G. M. et al. A prospecção tecnológica em energia e a pesquisa e desenvolvimento no Brasil: elementos de uma estratégia. Campinas: 2004. 13p. (Energy Discussion Paper).

KUPFER, D.; TIGRE, P. Prospecção tecnológica. In: CARUSO, L. A.; TIGRE, P. B. (Orgs.). Modelo SENAI de prospecção: documento metodológico. Montevideo: OIT/ CINTERFOR, 2004. 19p.
LIMA, S. M. V. et al. Projeto QUO

VADIS: o futuro da pesquisa agropecuária brasileira. Brasília: Embrapa Informação Tecnológica, 2005. 451p.

MACEDO, I. C. Estado da arte e tendências tecnológicas para energia. Brasília: Secretaria Técnica do Fundo Setorial de Energia: Centro de Gestão e Estudos Estratégicos, 2003. 76p. Disponível em: <http:www.cgee.org.br> Acesso em: 01 de set. de 2006.

PARIKKA, M. Global biomass fuel resources. Biomass and Bioenergy, v.27, p.613-620, Dec. 2004.

SIMIONI, F. J.; HOFF, D. N. Fatores críticos à produção florestal em Santa Catarina: um estudo de prospecção. In: CONGRESSO BRASILEIRO DE ECONOMIA E SOCIOLOGIA RURAL, 54., 2003, Fortaleza. Anais... Fortaleza: SOBER, 2006. p.1-15.

SIMIONI, F. J. et al. Análise das transações na cadeia produtiva de energia de biomassa de origem florestal. In: ENCONTRO NACIONAL DE ENGENHARIA DE PRODUÇÃO, 27., 2007, Foz do Iguaçú. Anais... Foz do Iguaçú: ENEGEP, 2007a.

SIMIONI, F. J. et al. Productive chain of forest biomass in south plateau of Santa Catarina. In: INTERNATIONAL PENSA CONFERENCE, 6., 2007, Ribeirão Preto. Anais... Ribeirão Preto: PENSA/ USP, 2007b.

WRIGHT, J. A técnica de Delphi. In: CASTRO, A. M. G.; COBBE, R. V.; GOEDERT, W. R. Prospecção de demandas tecnológicas: manual metodológico para o SNPA. Brasília: Embrapa, 1995. 82p. 